\title{
On the abstract characterization of quasi-varieties
}

\author{
M.C. Pedicchio E.M. Vitale
}

September 1999

\begin{abstract}
We show how the exact completion of a regular category constitutes a unifying framework for the abstract characterization of various classes of quasi-varieties.
\end{abstract}

\section{Introduction}

It is well known that a variety (in the classical sense of an HSP class of algebras) can be described in categorical terms as a category of algebras for a monad over SET, where the monad is finitary, i.e. it preserves filtered colimits. Varieties have been also characterized by Lawvere [16] (see theorem 2.3) as exact categories (in the sense of Barr [2]) with an abstractly finite, regular projective, regular generator.

Quasi-varieties are regular epireflective subcategories of varieties such that the inclusion functor is finitary. Recall that a full subcategory is regular epireflective when the inclusion has a left adjoint and units are regular epis, and that quasi-varieties correspond to classical SP classes of algebras closed under filtered colimits. Categorically, in a quasi-variety we cannot expect to get exactness any more, but we have to replace it with the weaker notion of regularity.

The aim of this note is to show that the characterization theorem for quasivarieties (corollary 4.6) can be easily deduced from the characterization theorem for varieties (theorem 2.3), using some very simple and general facts about the exact completion of a regular category. In section 3 we will recall the definition and basic properties of such a completion; then we will show how the same idea works also without any assumption of finiteness, that is for monadic categories over SET and their regular epireflective subcategories.

We will consider characterization theorems for the following five different kinds of categories:

- monadic categories over SET and, among them, varieties;

- (in decreasing order of generality) regular epireflective subcategories of monadic categories over SET, regular epireflective subcategories of varieties, quasi-varieties. 
Some known examples show that the various inclusions are strict:

i - compact Hausdorff spaces, sup-lattices, the category GR of grids and the category FR of frames are monadic over SET, but the monad is not finitary, so they are not varieties;

ii - the dual of the category of topological spaces is regular epireflective in GR and the dual of the category of sober spaces is regular epireflective in FR, but they are not regular epireflective in any variety [3];

iii - the groups which can be embedded in a product of countable groups form a regular epireflective subcategory of the variety of groups ; but this subcategory is not a quasi-variety since it is not closed under filtered colimits [1];

iv- a typical example of quasi-variety is the category of torsion-free abelian groups.

\section{Preliminaries}

In this section we recall the basic definitions we will use in the following, as well as the characterizations theorem for monadic categories over SET and for varieties. We also compare some notions of finiteness.

Our categories will always be regular, in the sense of Barr [2] ; we recall that a category is regular if it has finite limits, each arrow factors as a regular epi followed by a mono, and regular epis are pull-back stable. (It turns out that in a regular category the kernel pair of an arrow always has a coequalizer, given by the regular epi part of the factorization of the arrow. Notice that, unlike [2], in [4] the existence of all coequalizers is assumed. Since regular functors (see section 3 for the definition) do not preserve arbitrary coequalizers, we stick to the former definition.) A regular category is exact if each equivalence relation has a coequalizer and it is the kernel pair of its coequalizer.

To formulate the characterization theorem 2.1, we also need the following definitions : an object $P$ of a category $\mathbb{B}$ is regular projective if the representable functor $\mathbb{B}(P,-): \mathbb{B} \rightarrow$ SET preserves regular epis ; an object $P$ is a regular generator if, for any small set $S$, there exists the $S$-indexed copower $S \bullet P$ of $P$ and, moreover, for any object $X$ of $\mathbb{B}$ the canonical morphism $\mathbb{B}(P, X) \bullet P \rightarrow X$ is a regular epi. Now, we can state the following

Theorem 2.1 [9] A category $\mathbb{B}$ is equivalent to a monadic category over SET if and only if it is exact and it has a regular projective, regular generator $P$.

This theorem is due to Duskin [9]. It can be found in standard textbooks in category theory, e.g. [4] and [5] (see also [22]). To give an idea of the proof, we 
recall that for any object $P$ which has copowers indexed by small sets, we have two functors

$$
U: \mathbb{B} \longrightarrow \text { SET } U=\mathbb{B}(P,-) \text { and } F: \operatorname{SET} \longrightarrow \mathbb{B} F=-\bullet P
$$

such that $U$ is right adjoint to $F$. Then the functor

$$
T=F \cdot U=\mathbb{B}(P,-\bullet P): \mathrm{SET} \longrightarrow \mathrm{SET}
$$

is a monad over SET ; the hypotheses on $P$ in 2.1 imply that $\mathbb{B}$ is equivalent to the category of $T$-algebras. Observe that in this equivalence the object $P$ corresponds to the free algebra $F(1)$ on one generator.

Consider now the following definitions :

Definition 2.2 ([12], [5], [16]) Let $P$ be an object of a category $\mathbb{B}$;

$i-P$ is finitely presentable if the representable functor $\mathbb{B}(P,-)$ is finitary;

ii - $P$ is finitely generated if the representable functor $\mathbb{B}(P,-)$ preserves filtered unions (i.e. filtered colimits such that the canonical injections are monos);

iii - $P$ is abstractly finite if for any small set $S$ there exists the $S$-indexed copower $S \bullet P$ of $P$ and, moreover, any arrow $P \rightarrow S \bullet P$ factors through $S^{\prime} \bullet P$ for some finite subset $S^{\prime}$ of $S$.

Observe that an object $P$ is abstractly finite if and only if the functor $T=\mathbb{B}(P,-\bullet P):$ SET $\rightarrow$ SET induced by $P$ is finitary. In fact, if $\mathbb{B}(P,-\bullet P)$ preserves filtered colimits, then $P$ is abstractly finite since a set $S$ is the filtered union of its finite subsets. Conversely, it is known that a functor $T$ : SET $\rightarrow$ SET preserves filtered colimits iff it preserves filtered colimits of diagrams of monos, and this holds for $T=\mathbb{B}(P,-\bullet P)$ since $P$ is abstractly finite (see [8]).

This remark together with theorem 2.1 gives Lawvere characterization of varieties.

Theorem 2.3 [16] A category is equivalent to a variety, i.e. to a monadic category over SET for a finitary monad, if and only if it is exact and it has an abstractly finite, regular projective, regular generator $P$.

Remark: The passage from one-sorted varieties to many-sorted varieties corresponds to the passage from monads over SET to monads over a power of SET. The two previous theorems still hold provided that we replace the single generator by a small family of generators.

Observe that in a variety, the free algebra on one generator is finitely presentable. Consequently, in theorem 2.3 "abstractly finite" can be equivalently replaced by "finitely presentable". Dropping the assumption of exactness, this is 
no longer true, as attested by example (iii) in the introduction ( $\mathbb{Z}$ is abstractly finite but not finitely presentable in the quoted regular epireflective subcategory of the variety of groups) (see also [13]). The next proposition shows what remains true in general.

Proposition 2.4 Let $P$ be an object in a category $\mathbb{B}$.

$i$ - if $P$ is finitely generated and $P$ has copowers, then $P$ is abstractly finite;

ii - if $P$ is an abstractly finite, regular projective, regular generator, then $P$ is finitely generated.

Proof: i): let $S$ be a non-empty set ; $S$ is the filtered union of its non-empty finite subsets $S^{\prime}$. Since any inclusion $S^{\prime} \hookrightarrow S$ has a retraction, $S \bullet P$ is the filtered union of the various $S^{\prime} \bullet P$.

ii): first observe that any arrow $f: P \rightarrow \coprod_{I} A_{i}$ factors through a finite coproduct of the $A_{i}$ 's. In fact, since $P$ is a regular generator, for each element $i$ of $I$ there exists a regular epi $\sigma_{i}: S_{i} \bullet P \rightarrow A_{i}$. These various $\sigma_{i}$ 's give rise to a regular epi $\sigma=\coprod \sigma_{i}$ and, since $P$ is regular projective, $f$ factors through $\sigma$. Let $g: P \rightarrow\left(\coprod_{I} S_{i}\right) \bullet P$ be this factorization. Since $P$ is abstractly finite, $g$ factors through $S^{\prime} \bullet P$ for some finite subset $S^{\prime}$ of $\coprod_{I} S_{i}$. Since $S^{\prime}$ is finite, there are only finitely many elements $i$ in $I$ such that $S^{\prime} \cap S_{i}$ is not empty. Finally, $f$ factors through the coproduct of the $A_{i}$ 's indexed by those $i$.

Now, given a filtered colimit $\operatorname{colim} A_{i}$ and an arrow $h: P \rightarrow \operatorname{colim} A_{i}$, we can see colim $A_{i}$ as a regular quotient of a coproduct $\coprod_{I} A_{i}$. Since $P$ is regular projective, $h$ factors through some $f: P \rightarrow \coprod_{I} A_{i}$. By the previous argument, $f$ factors through a finite coproduct $A_{i_{1}}+\ldots+A_{i_{n}}$ and, by filteredness, through a single $A_{i_{0}}$. This shows that the canonical factorization $\operatorname{colim}\left(\mathbb{B}\left(P, A_{i}\right)\right) \rightarrow$ $\mathbb{B}\left(P, \operatorname{colim} A_{i}\right)$ is surjective. It is surely injective if the injections $A_{i} \rightarrow \operatorname{colim} A_{i}$ are monos, that is if $\operatorname{colim} A_{i}$ is a filtered union.

\section{The exact completion of a regular category}

We recall that the exact completion of a regular category $\mathbb{B}$ is defined as an exact category $\mathbb{B}_{\text {ex }}$ together with a regular (= finite limits and regular epimorphisms preserving) functor

$$
\gamma: \mathbb{B} \longrightarrow \mathbb{B}_{\mathrm{ex}}
$$

such that the following universal property holds :

for any exact category $\mathbb{C}$ and for any regular functor $F: \mathbb{B} \longrightarrow \mathbb{C}$ there exists a unique (up to natural isomorphisms) regular functor $\hat{F}: \mathbb{B}$ ex $\longrightarrow \mathbb{C}$ with $\gamma \cdot \hat{F} \simeq$ F 


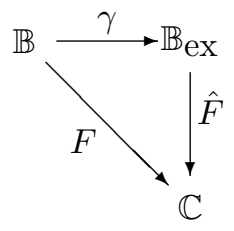

More precisely, composition with $\gamma$ induces an equivalence between the category of regular functors from $\mathbb{B}$ to $\mathbb{C}$ and the category of regular functors from $\mathbb{B}_{\mathrm{ex}}$ to $\mathbb{C}$. The construction of $\mathbb{B}_{\text {ex }}$ for a regular category $\mathbb{B}$ and its universal property are discussed in detail in [6], [7], [11] and [20]. Objects in $\mathbb{B}_{\mathrm{ex}}$ are equivalence relations in $\mathbb{B}$, and an arrow $\alpha:\left(r_{0}, r_{1}: R \rightrightarrows X\right) \rightarrow\left(s_{0}, s_{1}: S \rightrightarrows Y\right)$ in $\mathbb{B}_{\mathrm{ex}}$ is a relation from $X$ to $Y$ such that $R \cdot \alpha \cdot S=\alpha, \alpha^{o} \cdot \alpha<S$ and $\alpha \cdot \alpha^{o}>R$ (where the point - denotes the composition of relations and $\alpha^{o}$ the opposite of $\alpha$ ). For basic notions on relations and equivalence relations, see [5]. Composition in $\mathbb{B}_{\text {ex }}$ is the usual composition of relations of $\mathbb{B}$, and the identity of an object $\left(r_{0}, r_{1}: R \rightrightarrows X\right)$ is the relation itself.

In the next lemma we list the properties of the unit $\gamma: \mathbb{B} \rightarrow \mathbb{B}_{\text {ex }}$ needed to obtain the announced characterization of quasi-varieties. The first point is known and we omit the proof (see for example [20]) ; the second and the third points strengthen some facts already implicit in [6].

Lemma 3.1 Let $\mathbb{B}$ be a regular category and consider its exact completion $\gamma: \mathbb{B} \rightarrow \mathbb{B}_{e x}$

1) $\gamma$ is full, faithful and exact;

2) $\gamma$ preserves regular projective objects and their coproducts;

3) $\mathbb{B}$ is equivalent to a regular epireflective subcategory of $\mathbb{B}_{\text {ex }}$ iff $\gamma: \mathbb{B} \longrightarrow \mathbb{B}_{\text {ex }}$ has a left adjoint and units are regular epimorphisms iff $\mathbb{B}$ has coequalizers of equivalence relations.

Proof: 2): recall from [20] that, for each object $\left(r_{0}, r_{1}: R \rightrightarrows X\right)$ (which we write $\left.\left[r_{0}, r_{1}\right]\right)$ of $\mathbb{B}_{\mathrm{ex}}$, the following is an exact sequence

$$
\gamma(R) \underset{\gamma\left(r_{1}\right)}{\stackrel{\gamma\left(r_{0}\right)}{\longrightarrow}} \gamma(X) \stackrel{R}{\longrightarrow}\left[r_{0}, r_{1}\right]
$$

(that is, a kernel pair - coequalizer sequence). Now consider a regular projective object $P$ of $\mathbb{B}$ and a regular epi $\alpha:\left[r_{0}, r_{1}\right] \rightarrow \gamma(P)$; the composite $R \cdot \alpha$ is of the form $\gamma(f)$ for a unique $f: X \rightarrow P$. Since $\gamma$ is full, faithful and preserves finite limits, it reflects regular epis, so that $f$ is a regular epi and then it has a section $s: P \rightarrow X$. Finally, $\gamma(s) \cdot R$ is a section for $\alpha$. This implies that $\gamma(P)$ is regular projective, since in $\mathbb{B}_{\mathrm{ex}}$ regular epis are pull-back stable. 
Now, we have to prove that $\gamma$ preserves coproducts of regular projective objects. Consider a small family $\left(P_{i}\right)_{i \in I}$ of regular projective objects in $\mathbb{B}$ and let

$$
\left(\sigma_{i}: P_{i} \longrightarrow \coprod_{I} P_{i}\right)_{I}
$$

be their coproduct. Consider also an object $\left[r_{0}, r_{1}\right]$ in $\mathbb{B}_{\text {ex }}$ and a family of arrows

$$
\left(x_{i}: \gamma\left(P_{i}\right) \longrightarrow\left[r_{0}, r_{1}\right]\right)_{I} \text {. }
$$

Since $R: \gamma(X) \longrightarrow\left[r_{0}, r_{1}\right]$ is a regular epi and each $\gamma\left(P_{i}\right)$ is regular projective, we obtain a family of arrows

$$
\left(y_{i}: \gamma\left(P_{i}\right) \longrightarrow \gamma(X)\right)_{I}
$$

such that, for each $i$ in $I$, one has $y_{i} \cdot R=x_{i}$. Since $\gamma$ is full and faithful, for each $i$ in $I$ there exists a unique $z_{i}: P_{i} \longrightarrow X$ such that $\gamma\left(z_{i}\right)=y_{i}$. Since $\coprod_{I} P_{i}$ is a coproduct in $\mathbb{B}$, there exists a unique $z: \coprod_{I} P_{i} \longrightarrow X$ such that $\sigma_{i} \cdot z=z_{i}$ for each $i$. In this way we get an arrow $\gamma(z) \cdot R: \gamma\left(\coprod_{I} P_{i}\right) \longrightarrow\left[r_{0}, r_{1}\right]$ such that $\gamma\left(\sigma_{i}\right) \cdot \gamma(z) \cdot R=x_{i}$ for each $i$. It remains to prove that such a factorization $\gamma(z) \cdot R$ is unique. For this, suppose there are two arrows $f, g: \gamma\left(\coprod_{I} P_{i}\right) \longrightarrow\left[r_{0}, r_{1}\right]$ such that $\gamma\left(\sigma_{i}\right) \cdot f=x_{i}$ and $\gamma\left(\sigma_{i}\right) \cdot g=x_{i}$ for each $i$ in $I$. Since each $P_{i}$ is regular projective in $\mathbb{B}$, their coproduct $\coprod_{I} P_{i}$ is regular projective in $\mathbb{B}$, and then $\gamma\left(\coprod_{I} P_{i}\right)$ is regular projective in $\mathbb{B}_{\mathrm{ex}}$. We get then two factorizations $\bar{f}, \bar{g}: \gamma\left(\coprod_{I} P_{i}\right) \longrightarrow \gamma(X)$ such that $\bar{f} \cdot R=f$ and $\bar{g} \cdot R=g$. Now fix an $i$ in $I$ and observe that $\gamma\left(\sigma_{i}\right) \cdot \bar{f} \cdot R=\gamma\left(\sigma_{i}\right) \cdot f=x_{i}=\gamma\left(\sigma_{i}\right) \cdot g=\gamma\left(\sigma_{i}\right) \cdot \bar{g} \cdot R$. Since $\gamma\left(r_{0}\right), \gamma\left(r_{1}\right): \gamma(R) \longrightarrow \gamma(X)$ is the kernel pair of $R: \gamma(X) \longrightarrow\left[r_{0}, r_{1}\right]$, there exists a unique arrow $t_{i}: \gamma\left(P_{i}\right) \longrightarrow \gamma(R)$ such that $t_{i} \cdot \gamma\left(r_{0}\right)=\gamma\left(\sigma_{i}\right) \cdot \bar{f}$ and $t_{i} \cdot \gamma\left(r_{1}\right)=\gamma\left(\sigma_{i}\right) \cdot \bar{g}$. Once again, the arrow $t_{i}$ is $\gamma\left(\tau_{i}\right)$ for a unique $\tau_{i}: P_{i} \longrightarrow R$. From the family of arrows

$$
\left(\tau_{i}: P_{i} \longrightarrow R\right)_{I}
$$

we get a unique $\tau: \coprod_{I} P_{i} \longrightarrow R$ such that $\sigma_{i} \cdot \tau=\tau_{i}$ for each $i$. Now call $\tilde{f}, \tilde{g}: \coprod_{I} P_{i} \longrightarrow X$ the arrows such that $\gamma(\tilde{f})=\bar{f}$ and $\gamma(\tilde{g})=\bar{g}$ and observe that for each $i$ one has $\gamma\left(\sigma_{i} \cdot \tilde{f}\right)=\gamma\left(\sigma_{i}\right) \cdot \bar{f}=t_{i} \cdot \gamma\left(r_{0}\right)=\gamma\left(\tau_{i} \cdot r_{0}\right)=\gamma\left(\sigma_{i} \cdot \tau \cdot r_{0}\right)$. This implies that $\tilde{f}=\tau \cdot r_{0}$ because $\gamma$ is full and faithful and $\coprod_{I} P_{i}$ is a coproduct in $\mathbb{B}$. In the same way, $\tilde{g}=\tau \cdot r_{1}$. Finally, $f=\bar{f} \cdot R=\gamma(\tilde{f}) \cdot R=$ $\gamma(\tau) \cdot \gamma\left(r_{0}\right) \cdot R=\gamma(\tau) \cdot \gamma\left(r_{1}\right) \cdot R=\gamma(\tilde{g}) \cdot R=\bar{g} \cdot R=g$.

$3)$ : Clearly, if $\mathbb{B}$ is a regular epireflective subcategory of the exact category $\mathbb{B}_{\mathrm{ex}}$, then it has coequalizers of equivalence relations. The only non-trivial implication which remains to prove is that if $\mathbb{B}$ has coequalizers of equivalence relations, then $\gamma$ has a left adjoint and units are regular epimorphisms. Let $\left[r_{0}, r_{1}\right]$ be an object of $\mathbb{B}_{\text {ex }}$; by assumption, the coequalizer $q: X \rightarrow Q$ of the pair $r_{0}, r_{1}$ exists in $\mathbb{B}$. Since $\gamma\left(r_{0}\right) \cdot \gamma(q)=\gamma\left(r_{1}\right) \cdot \gamma(q)$, there exists a unique 
arrow $\eta_{\left[r_{0}, r_{1}\right]}:\left[r_{0}, r_{1}\right] \rightarrow \gamma(Q)$ such that $R \cdot \eta_{\left[r_{0}, r_{1}\right]}=\gamma(q)$. Moreover, since $\gamma$ preserves regular epis, $\eta_{\left[r_{0}, r_{1}\right]}$ is a regular epi. It remains to prove the universality of $\eta_{\left[r_{0}, r_{1}\right]}$. Let $\alpha:\left[r_{0}, r_{1}\right] \rightarrow \gamma(Y)$ be an arrow in $\mathbb{B}_{\text {ex }}$ and consider the composite $R \cdot \alpha$, which is of the form $\gamma(\epsilon)$ for $\epsilon: X \rightarrow Y$. Observe that $\gamma\left(r_{0}\right) \cdot R \cdot \alpha=\gamma\left(r_{1}\right) \cdot R \cdot \alpha$, so that $r_{0} \cdot \epsilon=r_{1} \cdot \epsilon$ and then there exists $\epsilon^{\prime}: Q \rightarrow Y$ such that $q \cdot \epsilon^{\prime}=\epsilon$. Since $R$ is a (regular) epi, this implies that $\eta_{\left[r_{0}, r_{1}\right]} \cdot \gamma\left(\epsilon^{\prime}\right)=\alpha$. This factorization is certainly unique because $\eta_{\left[r_{0}, r_{1}\right]}$ is an epi and $\gamma$ is faithful.

\section{Characterization of quasi-varieties}

Proposition 4.1 Let $\mathbb{B}$ be a regular category with a regular projective, regular generator $P$; then $\mathbb{B}_{\text {ex }}$ is equivalent to a monadic category over SET.

Proof: The category $\mathbb{B}_{\mathrm{ex}}$ is exact and, by part 2 of lemma $3.1, \gamma(P)$ is regular projective. To show that $\mathbb{B}_{\mathrm{ex}}$ satisfies all the conditions of theorem 2.1, it remains to prove that $\gamma(P)$ is a regular generator. For this, let $\left[r_{0}, r_{1}\right]=$ $\left(r_{0}, r_{1}: R \rightrightarrows X\right)$ be an object of $\mathbb{B}_{\mathrm{ex}}$ and consider the following commutative diagram

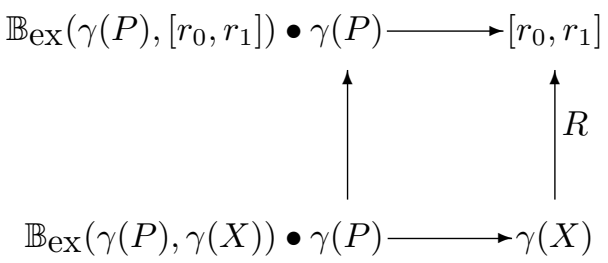

where the left-hand arrow is induced by composition with $R$. The vertical arrow $R$ is a coequalizer. Moreover by 1 and 2 of lemma 3.1, the bottom arrow is a regular epi since it is $\gamma$ of the regular epi $\mathbb{B}(P, X) \bullet P \longrightarrow X$, then the top arrow is a regular epi. (The coproducts of $\gamma(P)$ involved in the previous diagram exist, by point 2 of lemma 3.1 , because they are indexed by small sets : in fact $\mathbb{B}$ is regular with a regular generator so that, by proposition 4.5 .15 in $[5], \mathbb{B}_{\text {ex }}$ is locally small.)

Corollary 4.2 Let $\mathbb{B}$ be a category ; the following conditions are equivalent:

$1) \mathbb{B}$ is a regular category with a regular projective, regular generator $P$ and, moreover, it has coequalizers of equivalence relations;

2) $\mathbb{B}$ is equivalent to a regular epireflective subcategory of a monadic category over SET. 
Proof: 2$) \Rightarrow 1$ ) is standard (take as $P$ the reflection of the free algebra on one generator). 1$) \Rightarrow 2$ ): by part 3 of lemma $3.1, \mathbb{B}$ is a regular epireflective subcategory of $\mathbb{B}_{\mathrm{ex}}$, so we can conclude by proposition 4.1 .

Proposition 4.3 Let $\mathbb{B}$ be a regular category with an abstractly finite, regular projective, regular generator $P$; then $\mathbb{B}_{e x}$ is equivalent to a variety.

Proof: By part 2 of lemma 3.1, the condition to be abstractly finite is preserved by $\gamma$, so that $\mathbb{B}_{\text {ex }}$ satisfies all the conditions of theorem 2.3 : hence $\mathbb{B}_{\text {ex }}$ is a variety.

Corollary 4.4 Let $\mathbb{B}$ be a category ; the following conditions are equivalent:

1) $\mathbb{B}$ is a regular category with an abstractly finite, regular projective, regular generator $P$ and, moreover, it has coequalizers of equivalence relations;

2) $\mathbb{B}$ is equivalent to a regular epireflective subcategory of a variety.

Proof: 2$) \Rightarrow 1$ ): to prove that the reflection in $\mathbb{B}$ of the free algebra on one generator is abstractly finite, one uses the regular projectivity of the free algebra on one generator. 1$) \Rightarrow 2$ ): it follows from proposition 4.3 and part 3 of lemma 3.1 .

Proposition 4.5 Let $\mathbb{B}$ be a regular category with a finitely presentable, regular projective, regular generator $P$; then $\mathbb{B}_{e x}$ is equivalent to a variety and $\gamma: \mathbb{B} \rightarrow \mathbb{B}_{\text {ex }}$ is finitary.

Proof: Consider the following commutative diagram

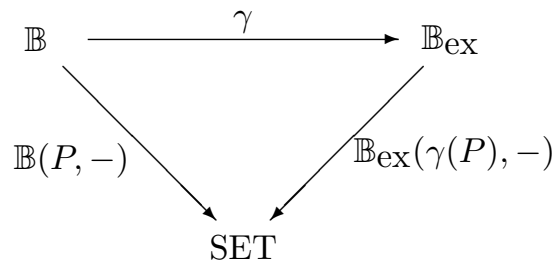

Since finitely presentable implies abstractly finite, we already know that $\mathbb{B}_{\text {ex }}$ is equivalent to a variety. In this equivalence $\gamma(P)$ corresponds to the free algebra on one generator, so that $\mathbb{B}_{\operatorname{ex}}(\gamma(P),-)$ reflects filtered colimits. Then $\gamma$ preserves filtered colimits since, by assumption, $\mathbb{B}(P,-)$ preserves them. 
Corollary 4.6 Let $\mathbb{B}$ be a category; the following conditions are equivalent:

1) $\mathbb{B}$ is a regular category with a finitely presentable, regular projective, regular generator $P$ and, moreover, it has coequalizers of equivalence relations;

2) $\mathbb{B}$ is equivalent to a regular epireflective subcategory of a variety, and the inclusion functor is finitary, i.e. $\mathbb{B}$ is equivalent to a quasi-variety.

Proof: 2) $\Rightarrow 1$ ): if the right adjoint preserves filtered colimits, then the left adjoint sends finitely presentable objects to finitely presentable objects. 1) $\Rightarrow 2$ ): by proposition $4.5, \mathbb{B}_{\mathrm{ex}}$ is a variety and $\gamma$ is finitary. Moreover, by part 3 of lemma $3.1, \mathbb{B}$ is a regular epireflective subcategory of $\mathbb{B}_{\mathrm{ex}}$.

Remarks: 1) once again, the passage from monads over SET to monads over a power of SET (or from one-sorted varieties to many-sorted varieties) is straightforward: it is enough to replace the single generator by a small family of generators.

2) The characterization of regular epireflective subcategories of varieties given in corollary 4.4 is due to Isbell [14]. The case of regular epireflective subcategories of monadic categories comes back to Felscher [10], Keane [15] and Tholen [21]; a proof which uses the exact completion of a weakly left exact category is sketched in [23]. Corollary 4.2 also appears in [18] where the case of $k$-permutable quasivarieties is considered.

3) Asking, in propositions 4.1, 4.3 and 4.5 , that $\mathbb{B}$ is preadditive, one has that $\mathbb{B}_{\text {ex }}$ is abelian. But abelian varieties are exactly module categories over unital rings (see [16]). For this reason, adding preadditivity on $\mathbb{B}$, corollaries 4.4 and 4.6 give characterizations of (regular) epireflective subcategories of module categories.

Acknowledgements. We would like to thank J. Adamek and the anonymous referee for some useful comments on an earlier version of this work.

\section{5}

\section{References}

[1] J. AdameK, J. Rosicky: Locally presentable and accessible categories, Cambridge University Press (1994).

[2] M. Barr, P. GRILlet AND D. VAn Osdol: Exact categories and categories of sheaves, Springer LNM 236 (1971).

[3] M. BARr, M.C. Pedicchio: Topological spaces and quasi-varieties, Appl. Categ. Struct. 4 (1996) 81-85. 
[4] M. Barr And C. Wells: Toposes, triples and theories, Springer (1985).

[5] F. Borceux: Handbook of categorical algebra, vol. 1 and 2, Cambridge University Press (1994).

[6] A. Carboni and E.M. Vitale: Regular and exact completions, J. Pure Appl. Algebra 125 (1998) 79-116.

[7] A. Carboni and R.F.C. Walters: Cartesian bicategories I, J. Pure Appl. Algebra 49 (1987) 11-32.

[8] B. DAY: Note on monoidal localizations, Bull. Austral. Math. Soc. 8 (1973) 1-16.

[9] J. Duskin: Variations on Beck's tripleability theorem, Springer LNM 106 (1969) 74-129.

[10] W. FELSCHER: Kennzeichnung von primitiven und quasiprimitiven Kategorien von Algebren, Arch. Math. (Basel) 39 (1968) 390-397.

[11] P. Freyd And A. Scedrov: Categories, allegories, North-Holland (1990)

[12] P. Gabriel, F. Ulmer: Lokal prsentierbare Kategorien, Springer LNM 221 (1971).

[13] H. Herrlich: A characterization of k-ary algebraic categories, Manuscripta Math. 4 (1971) 277-284.

[14] J.R. IsBELL: Subobjects, adequacy, completeness and categories of algebras, Rozprawy Mat. 36 (1964) 33 p.

[15] O. Keane: Abstract Horn theories, Springer LNM 445 (1975) 15-50.

[16] F.W. Lawvere: Functorial semantics of algebraic theories, Proc. Nat. Acad. Sc. USA 50 (1963) 869-872.

[17] P. Linton Some aspects of equational categories, Proc. Conf. Categor. Algebra, La Jolla 1965, Springer (1966) 84-94.

[18] M.C. Pedicchio: On k-permutability for categories of T-algebras, Logic and algebra, Marcel Dekker LN Pure Appl. Math. 180 (1996) 637-646.

[19] M.C. Pedicchio AND J. Rosicky: Localizations of varieties and quasivarieties, preprint (1998). 
[20] R. Succi Cruciani: La teoria delle relazioni nello studio di categorie regolari e di categorie esatte, Riv. Mat. Univ. Parma (4) 1 (1975) 143158.

[21] W. Tholen: Relative Bildzerlegungen und algebrische Kategorien, Ph.D. Thesis, Munster (1974).

[22] E.M. ViTALE: On the characterization of monadic categories over Set, Cahiers de Topologie et Gom. Diff. Catgoriques XXXV (1994) 351-358.

[23] E.M. Vitale: Localizations of algebraic categories, J. Pure Appl. Algebra 108 (1996) 315-320.

Maria Cristina Pedicchio :

Dipartimento di Matematica, Universit di Trieste,

P.le Europa 1, 34100 Trieste, Italy.

e.mail : pedicchi@univ.trieste.it

Enrico M. Vitale :

Dpartement de Mathmatique, Universit catholique de Louvain, Chemin du Cyclotron 2, 1348 Louvain-la-Neuve, Belgium.

e.mail : vitale@agel.ucl.ac.be 